\title{
THE DEVELOPMENT OF THE TORUN VLBI STATION
}

\author{
Stanislaw Gorgolewski \\ Torun Radio Astronomy Observatory \\ Nicolaus Copernicus University \\ Torun, Poland
}

\begin{abstract}
The Torun VLBI Station is situated in Piwnice near the site of the Torun Radio Astronomical Observatory on the Nicolaus Copernicus University Land Estate, about $10 \mathrm{~km}$ north of Torun. The VLBI station development started in 1976, when the rubidium frequency standard was ordered and the ground was broken for the foundation of the 15-m equatorially mounted dish. The construction of the Mark II-C VLBI system began in 1977, and the completed VLBI station was first tested in 1980. The first fringes were obtained on May 31, 1981, with the Effelsberg 100-m dish. This experiment started our work with the European VLBI Network (EVN), and later in 1984 with the Global VLBI Network. We now have five radiometers and have received funding for the construction of a new VLBI station, with a 32-m dish, which is now in the final design stage.
\end{abstract}

\section{INTRODUCTION}

The Torun VLBI team is an outgrowth from our first radio astronomical research dating from 1958 in the field of monitoring of the Sun during the IGY. Two British Council scholarships for research students at Cavendish Laboratory in Cambridge, England, brought us into the field of solar coronal investigations and radio source structure studies. This research involved the use of interferometric techniques and was the stepping stone to VLBI. We began using VLBI after several years of intense instrumental work that was supported by the European and the U.S. VLBI communities.

\section{MATERIALS AND METHODS}

The first VLBI radiometers came from the Westerbork Synthesis Radio Telescope $(50$ and $6 \mathrm{~cm}$ ) and the Max Planck Institut für Radioastronomie $(2.8 \mathrm{~cm})$. New local developments were the 18,92 , and $21 \mathrm{~cm}$ radiometers as well as the data quality analyzer/decoder and coherence test systems for use at all VLBI bands covered in Torun, i.e., $6,18,21,50$, and $92 \mathrm{~cm}$ wavelengths. The $15-\mathrm{m}$ dish, designed with generous Westerbork advice and experience, was built in Poland. The computer control system of the 15-m telescope underwent extensive modernization. It is now automatically running the VLBI observing. It is also used to plot the source 
maps from the data obtained from another computer that analyzes the correlated signals after primary data reduction in Bonn or Caltech.

The new VLBI project, to be completed by 1990, involves the construction of a new $32-\mathrm{m}$ precision radio telescope and the acquisition of a hydrogen maser frequency standard and a VLBA compatible recording system. The funding of this enterprise started in 1986 and enabled us to nearly complete the final design of the 32-m dish and to obtain a PDP-11/45 look-alike as well as two IBM PC-compatible computers. The 32-m telescope is needed to ensure a far better sensitivity than that of the 15-m dish. It shall also be useful for the QUASAT and RADIOASTRON missions, thus enabling us to make another important step into space VLBI. The 32-m dish will also be used with the existing 15-m dish for a local interferometer on a 200-m E-W baseline.

\section{ACKNOWLEDGMENTS}

The development of the Torun VLBI station was the result of nearly 10 years of concentrated effort of our group, which had nearly 20 years of previous experience. The first impulse for radio astronomy in Torun came from Prof. W. Iwanowska, who in turn was inspired by Martin Ryle, whose genius she had already recognized in the mid-fifties. Capital investment in the Torun Radio Astronomy Observatory (TRAO) has been provided by the Ministry of Science and Higher Education through the Nicolaus Copernicus University. Operational costs of the VLBI projects were shared by the university, the Satellite Geodesy Division of the Space Research Centre, and the Copernicus Astronomical Centre, both in Warsaw. Crucial instrumental, educational, and technical support has been generously provided by the European and U.S. communities. Without this support, we would never have been able to begin VLBI work in the early eighties if ever at all. This work was made possible by the generous support from the I.A.U., which awarded me a travel grant within the U.S. and subsistence for participation during I.A.U. Symposium 129. Travel expenses to the U.S. and back to Poland as well as the support for our research in this field since 1986 have been borne by the "Resortowy Program Badan Rozwojowych" (The Ministry of Science and Higher Education): RPBR NR. RR. I. 11/2. 